\title{
Trefoil factor 3 expression in epithelial ovarian cancer exerts a minor effect on clinicopathological parameters
}

\author{
FRIEDERIKE HOELLEN $^{1}$, ATHINA KOSTARA ${ }^{1}$, THOMAS KARN ${ }^{2}$, UWE HOLTRICH ${ }^{2}$, \\ AHMED EL-BALAT $^{2}$, MIKE OTTO $^{3}$, ACHIM RODY $^{1}$ and LARS C. HANKER ${ }^{1}$ \\ ${ }^{1}$ Department of Gynecology and Obstetrics, University of Lübeck, D-23538 Lübeck; \\ ${ }^{2}$ Department of Obstetrics and Gynecology, Goethe University, D-60323 Frankfurt; ${ }^{3}$ Medical Center \\ for Histopathology, Cytology and Molecular Diagnostics, D-54296 Trier, Germany
}

Received April 21, 2016; Accepted August 8, 2016

DOI: $10.3892 / \mathrm{mco} .2016 .994$

\begin{abstract}
The role of trefoil factor 3 (intestinal) (TFF3) has been analyzed in numerous cancers, such as breast and gastrointestinal cancer, and has been associated with poor prognosis. However, the role of TFF3 in ovarian cancers is not clear. Expression analysis of TFF3 in 91 ovarian cancer patients was performed by immunohistochemistry of primary paraffin-embedded tumor samples. The results were scored according to staining intensity and percentage of positive tumor cells resulting in an immune-reactive score (IRS) of 0-12. These results were correlated with clinicopathological characteristics and survival. TFF3 expression in our patient cohort exhibited a tendency towards improved overall and progression-free survival (PFS). In TFF3-positive serous and high-grade serous ovarian cancers, the median PFS was 27.6 months [95\% confidence interval (CI): 0-55.7] vs. 15.2 months in TFF3-negative tumors (95\% CI: 13.8-16.6) $(\mathrm{P}=0.183)$. The median overall survival was 53.9 months in TFF3-positive tumors (95\% CI: Non-applicable) vs. 44.4 months in TFF3-negative cases (95\% CI: 30.5-58.3) $(\mathrm{P}=0.36)$. TFF3 negativity was significantly associated with higher tumor grade $(\mathrm{P}=0.05)$. Based on our results, further studies are required in order to elucidate whether survival and chemosensitivity are affected by TFF3 expression in ovarian cancer.
\end{abstract}

\section{Introduction}

Epithelial ovarian cancer (EOC) is the fifth most common lethal cancer in women. The majority of the patients present with advanced-stage disease and, therefore, an unfavorable

Correspondence to: Dr Lars C. Hanker, Department of Gynecology and Obstetrics, University of Lübeck, 160 Ratzeburger Allee, D-23538 Lübeck, Germany

E-mail: lars.hanker@uksh.de

Key words: trefoil factor 3, ovarian cancer, chemosensitivity, platinum, trefoil factors prognosis (1). EOC is not a single disease entity. Histological subtyping is a reasonable first stratification; however, the identification of prognostic and predictive biomarkers remains a major challenge in order to establish targeted therapies. The five major histological/morphological types of ovarian carcinoma are as follows: High-grade serous carcinoma (68\%), clear cell $(12 \%)$, endometrioid (11\%), mucinous (3\%) and low-grade serous carcinoma (3\%) (2).

Despite high response rates to first-line chemotherapy, resistance to treatment frequently develops. Indeed, $~ 80 \%$ of the patients with International Federation of Gynecology and Obstetrics (FIGO) stage II-IV EOC will progress during or after adjuvant chemotherapy (3). Therefore, there is a need for new therapeutic approaches, as well as prognostic and predictive markers. Identification of new prognostic factors may help to distinguish different biological subgroups. In EOC, this is particularly important for the group of patients who develop recurrent disease. These patients often do not benefit from current treatment modalities and may suffer from severe therapy-related side effects. Future research should be focused on establishing more targeted and individualized treatment strategies in biologically distinct subgroups.

Trefoil factor 3 (TFF3) is an estrogen-regulated oncogene (4). TFF3 expression has been found to be associated with prognostic factors in a multitude of different types of cancer, including estrogen receptor-positive breast cancer (4). Furthermore, TFF3 has been found to be involved in gastric cancer progression (5). TFF3 is part of the marker panel that was selected for the calculator for ovarian carcinoma subtype prediction, introduced by Kölbel et al in 2011, as being most predictive of ovarian carcinoma subtype in both the archival and the tumor bank cohorts (2). The selection of TFF3 was based on comprehensive gene expression profiling data $(2,6)$. To the best of our knowledge, data on TFF3 in ovarian cancer are scarce. Therefore, the expression of the TFF3 in 91 ovarian cancer patients was investigated to determine its effect on prognosis and platinum sensitivity in ovarian cancer.

\section{Patients and methods}

Patients and treatment. The study included patients with primary EOC treated between 1995 and 2008 at 
the Department of Obstetrics and Gynecology of Goethe University (Frankfurt, Germany). A total of 91 patients were retrospectively analyzed. Formalin-fixed, paraffin-embedded (FFPE) tissue samples were obtained from the Department of Pathology. The patient characteristics are listed in Table I. Clinical and pathological factors were evaluated by reviewing medical charts and pathology records. The Local Research Ethics Committee approved studies of human tissue and the samples were processed anonymously. Clinical outcome was assessed from the date of surgery to the date of death or until the end of 2009. Only patients with histologically proven EOC were included. The majority of the patients had advanced-stage disease (FIGO III-IV). All the patients received primary surgery followed by platinum- and taxane-based chemotherapy.

Tissue samples and immunohistochemistry. The tissue samples were processed as previously described (7). Routine histopathological sections stained with hematoxylin and eosin were used for primary diagnosis and second reviewing (M.O.). Diagnosis and grading were performed according to the current World Health Organization criteria $(8,9)$. Following mounting on Superfrost Plus slides (Fisherbrand, Fisher Scientific, Hampton, NH, USA), 2- $\mu$ m paraffin sections were dewaxed in xylene and rehydrated with graduated ethanol treatment. For antigen retrieval, the sections were incubated for $20 \mathrm{~min}$ in a microwave oven $(800 \mathrm{~W})$ using citrate buffer $(10 \mathrm{mM}$; $\mathrm{pH}$ 6.0). The monoclonal anti-TFF3 antibody (ab57752, Lot GR71649-1; Abcam, Cambridge, UK,) was used at a dilution of 1:100. Incubation with the antibody for $1 \mathrm{~h}$ at room temperature was performed. For negative controls, the primary antibody was omitted. For secondary antibody incubation, the Dako REAL Detection System Alkaline Phosphatase/RED (REF K5005, Lot 20023341; Dako, Glostrup, Denmark) was applied, according to the manufacturer's instructions. The sections were counterstained with hematoxylin (Gill no. 3, Lot 060M4356; Sigma-Aldrich, St. Louis, Missouri, USA). Expression levels for cytoplasmic TFF3 were scored semi-quantitatively based on the product of staining intensity (SI) and percentage of positive cells (PP) using the immunoreactive score (IRS) as follows (10): IRS=SIxPP. SI was assigned as 0, negative; 1, weak; 2 , moderate; or 3 , strong. PP was defined as 0 , negative; $1,<10 \% ; 2,11-50 \% ; 3,51-80 \%$; or $4,>80 \%$ positive cells. All assessments were performed blinded with respect to clinical patient data.

Statistical analysis. For statistical analysis a cut-off value was defined according to the IRS, i.e., scores of 0-3 (negative and low) were collectively defined as a low score, whereas scores $\geq 3$ were defined as a high TFF3 expression score. The Chi-square and Fisher's exact tests were used to assess the associations between TFF3 expression of tumors and clinicopathological parameters. For those patients with available follow-up data, Kaplan-Meier curves were constructed and the log-rank test was used to determine the univariate significance of the variables. Cox regression analysis was performed to determine hazard ratios. All reported P-values were two-sided and P-values of $\leq 0.05$ were considered to indicate statistically significant differences. All analyses were performed using the SPSS software package, version 22.0 (IBM SPSS, Armonk, NY, USA).
Table I. Patients characteristics.

\begin{tabular}{|c|c|c|}
\hline Characteristics & $\begin{array}{l}\text { Number } \\
\text { (n) }\end{array}$ & $\%$ \\
\hline \multicolumn{3}{|l|}{ Age, years } \\
\hline$>50$ & 74 & 81.3 \\
\hline$\leq 50$ & 17 & 18.6 \\
\hline \multicolumn{3}{|l|}{ Grade } \\
\hline G3 & 62 & 68.1 \\
\hline $\mathrm{G} 1$ and $\mathrm{G} 2$ & 29 & 31.9 \\
\hline \multicolumn{3}{|l|}{ FIGO stage } \\
\hline $\mathrm{I}$ & 15 & 16.5 \\
\hline II & 7 & 8.2 \\
\hline III & 54 & 58.8 \\
\hline IV & 15 & 16.5 \\
\hline \multicolumn{3}{|c|}{ Histological subtype } \\
\hline Serous & 70 & 76.9 \\
\hline Other & 21 & 23.1 \\
\hline \multicolumn{3}{|c|}{ Residual tumor, cm } \\
\hline 0 & 43 & 47.3 \\
\hline $0-1$ & 19 & 20.9 \\
\hline$>1$ & 29 & 31.9 \\
\hline \multicolumn{3}{|c|}{ Platinum sensivity } \\
\hline Sensitive & 55 & 60.4 \\
\hline Resistant & 32 & 35.2 \\
\hline Not applicable & 4 & 4.4 \\
\hline \multicolumn{3}{|c|}{ Progression-free survival, months } \\
\hline Median & & 16.9 \\
\hline Range & & $14.0-19.7$ \\
\hline \multicolumn{3}{|c|}{ Overall survival, months } \\
\hline Median & & 51.6 \\
\hline Range & & $42.9-60.2$ \\
\hline
\end{tabular}

FIGO, international federation of obstetrics and gynecology.

\section{Results}

Patient characteristics. A total of 91 patients were included in this study. All the patients underwent primary debulking surgery including hysterectomy, bilateral salpingo-oophorectomy, infragastric omentectomy, appendectomy (mucinous subtype), systematic pelvic and para-aortic lymphadenectomy and, in distinct cases, peritonectomy and resection of affected tissues with intended resection of all visible tumors. In the majority of the patients $(n=62 ; 68 \%)$ optimal debulking, i.e., achieving an optimal postoperative residual tumor of $\leq 1 \mathrm{~cm}$, according to the National Comprehensive Cancer Network guidelines (11), could be achieved.

The median follow-up time was 33 months. The Kaplan-Meier estimate for median progression-free survival (PFS) for the entire group was 16.9 months [95\% confidence interval (CI): 14.0-19.7] and for overall survival (OS) 51.6 months (95\% CI: 42.9-60.2). The corresponding 5-year PFS and OS rates were $17.9 \pm 5.1$ and $34.9 \pm 6.2 \%$, respectively. In 
Table II. Clinical characteristics according to TFF3 expression.

\begin{tabular}{|c|c|c|c|c|}
\hline Characteristics & $\begin{array}{l}\text { TFF3-negative } \\
\text { (IRS 0-3); n (\%) }\end{array}$ & $\begin{array}{l}\text { TFF3-positive } \\
\text { (IRS 4-12); n (\%) }\end{array}$ & P-value & $\begin{array}{c}\text { Total } \\
(n=91)\end{array}$ \\
\hline Residual tumor, cm & & & 0.64 & \\
\hline 0 & $30(69.8)$ & $13(30.2)$ & & 43 \\
\hline$>0$ & $36(75.0)$ & $12(25.0)$ & & 48 \\
\hline Grade & & & 0.05 & \\
\hline 1,2 & $17(58.6)$ & $12(41.4)$ & & 29 \\
\hline 3 & $49(79.0)$ & $13(21.0)$ & & 62 \\
\hline Age, years & & & 0.77 & \\
\hline$>50$ & $53(71.6)$ & $21(28.4)$ & & 74 \\
\hline$\leq 50$ & $13(76.5)$ & $4(23.5)$ & & 17 \\
\hline FIGO stage & & & 0.29 & \\
\hline I, II & $14(63.6)$ & $8(36.4)$ & & 22 \\
\hline III, IV & $52(75.4)$ & $17(24.6)$ & & 69 \\
\hline Histological subtype & & & 0.01 & \\
\hline Serous & $56(80)$ & $14(20)$ & & 70 \\
\hline Other & $10(47.6)$ & $11(52.4)$ & & 21 \\
\hline Platinum response & & & n.a. & \\
\hline Sensitive & $39(70.9)$ & $16(29.1)$ & & 55 \\
\hline Resistant & $24(75.0)$ & $8(25.0)$ & & 32 \\
\hline Unknown/n.a. & $3(75.0)$ & $1(25.0)$ & & 4 \\
\hline
\end{tabular}

TFF3, trefoil factor 3; FIGO, international federation of obstetrics and gynecology; IRS, immunoreactive score; n.a., not applicable.

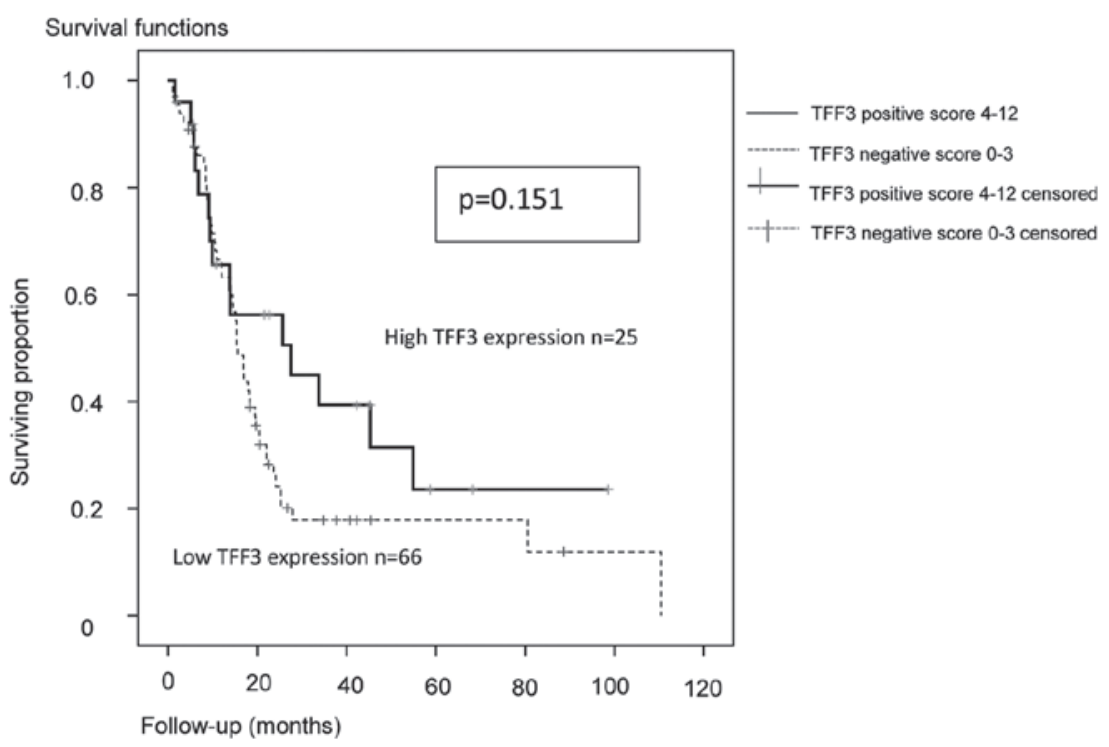

Figure 1. Kaplan-Meier analyses of progression-free survival was performed for all 91 patients with ovarian cancer according to TFF3 expression (TFF3-positive vs. -negative). TTF3, trefoil factor 3.

the group of serous ovarian cancers, the PFS was 15.4 months (95\% CI: 13.7-17.0) and the OS was 44.4 months (95\% CI: 26.0-62.1). The patient characteristics for the entire cohort are displayed in Table I.

TFF3 expression according to different clinicopathological characteristics. Immunohistochemistry revealed higher expression levels of TFF3 (IRS score $\geq 4$ ) in 25 tumor samples $(27.5 \%)$. In the cohort of 91 patients, no significant difference in TFF3 expression was found based on age, FIGO stage or residual tumor. In the group of serous carcinomas, there was a significant association with lower expression of TFF3 when compared with other subtypes. Furthermore, a significant correlation of TFF3 expression and grade was observed. 


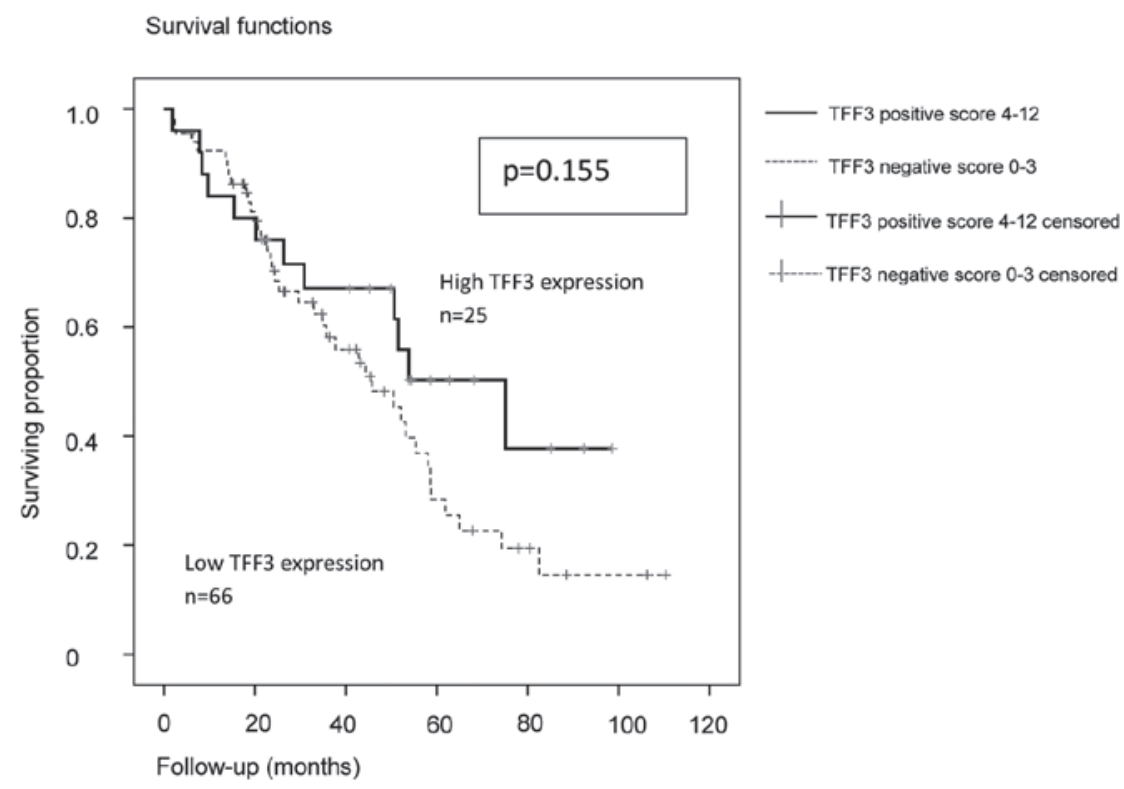

Figure 2. Kaplan-Meier analyses of overall survival was performed for all 91 patients with ovarian cancer according to TFF3 expression (TFF3-positive vs. -negative). TTF3, trefoil factor 3 .

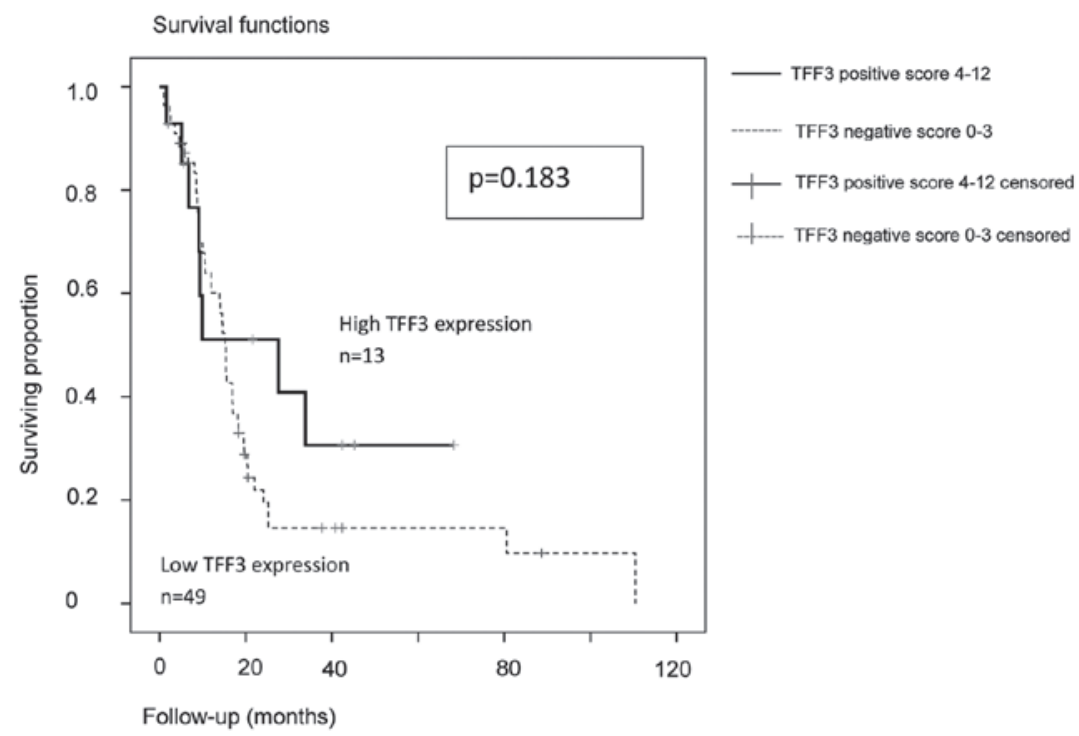

Figure 3. Kaplan-Meier analyses of progression-free survival was performed for the subgroup of 62 patients with high-grade serous ovarian cancer according to TFF3 expression (TFF3-positive vs. -negative). TFF3, trefoil factor 3.

Among TFF3-negative tumors (IRS=0-3), 25.8\% were G1 or G2 and $74.2 \%$ were G3, while among TFF3-positive tumors, grade was equally distributed between the two groups (48 vs. $52 \%$, respectively). The correlation of TFF3 negativity with higher grade was statistically significant $(\mathrm{P}=0.05$ Fisher's exact test; Table II).

Association of TFF3 expression with survival. In the Kaplan-Meier analysis of the entire cohort, the median PFS and OS were increased in TFF3-positive patients (Figs. 1 and 2). However, this was statistically not significant ( $\mathrm{P}=0.151$ and $\mathrm{P}=0.155$, respectively; log-rank Mantel-Cox) although there was a trend for a better PFS.

In the entire group, the median PFS in TFF3-positive patients was 27.6 months (95\% CI: 1.4-53.8) vs. 15.6 months
(95\% CI: 13.6-17.6) in TFF3-negative patients. The OS for the entire group in TFF3-positive patients was 75.1 months (95\% CI: 40.4-109.8) vs. 45.8 months (95\% CI: 30.5-61.6) in TFF3-negative cases.

By analyzing the subgroups of serous $(n=70)$ and high-grade serous $(n=62)$ ovarian carcinomas, the trend for a better PFS and OS in patients with high expression of TFF3 was confirmed. For the subgroup of high-grade serous ovarian cancer $(n=62)$, the PFS $(P=0.183)$ and OS $(P=0.36)$ results are shown in Figs. 3 and 4: In TFF3-positive serous and high-grade serous ovarian cancers, the median PFS was 27.6 months (95\% CI: 0-55.7) vs. 15.2 months in TFF3-negative cases (95\% CI: 13.8-16.6). The median OAS was 53.9 months in TFF3-positive (95\% CI: Non-applicable) vs. 44.4 months in TFF3-negative cases (95\% CI: 30.5-58.3). 
Table III. Univariate Cox regression analysis of overall and progression-free survival.

A, Overall survival

\begin{tabular}{|c|c|c|c|c|}
\hline Variables & P-value & HR & \multicolumn{2}{|c|}{$95 \%$ CI } \\
\hline Histology, other vs. serous & 0.102 & 0.548 & 0.266 & 1.126 \\
\hline Residual tumor, $\geq 0$ vs. $0 \mathrm{~cm}$ & 0.000 & 3.714 & 2.010 & 6.865 \\
\hline FIGO stage, III and IV vs. I and II & 0.001 & 1.085 & 1.036 & 1.137 \\
\hline TFF3-positive (IRS 4-12) vs. -negative (IRS 0-3) & 0.158 & 0.626 & 0.327 & 1.200 \\
\hline Age,$\leq 50$ vs. $>50$ years & 0.079 & 0.504 & 0.234 & 1.084 \\
\hline Grade, G3 vs. G1 and G2 & 0.141 & 0.949 & 0.884 & 1.018 \\
\hline
\end{tabular}

B, Progression-free survival

\begin{tabular}{lrrr}
\hline Variables & P-value & HR & 95\% CI \\
\hline Histology, other vs. serous & 0.043 & 0.531 & 0.288 \\
Residual tumor, $\geq 0$ vs. 0 cm & $<0.001$ & 3.165 & 0.981 \\
FIGO stage, III and IV vs. I and II & $<0.001$ & 1.065 & 5.308 \\
TFF3-positive (IRS 4-12) vs. -negative (IRS 0-3) & 0.156 & 0.654 & 1.031 \\
Age, $\leq 50$ vs. >50 years & 0.445 & 0.775 & 0.364 \\
Grade, G3 vs. G1 and G2 & 0.095 & 0.949 & 0.403 \\
\hline
\end{tabular}

TFF3, trefoil factor 3; FIGO, international federation of obstetrics and gynecology; HR, hazard ratio; CI, confidence interval.

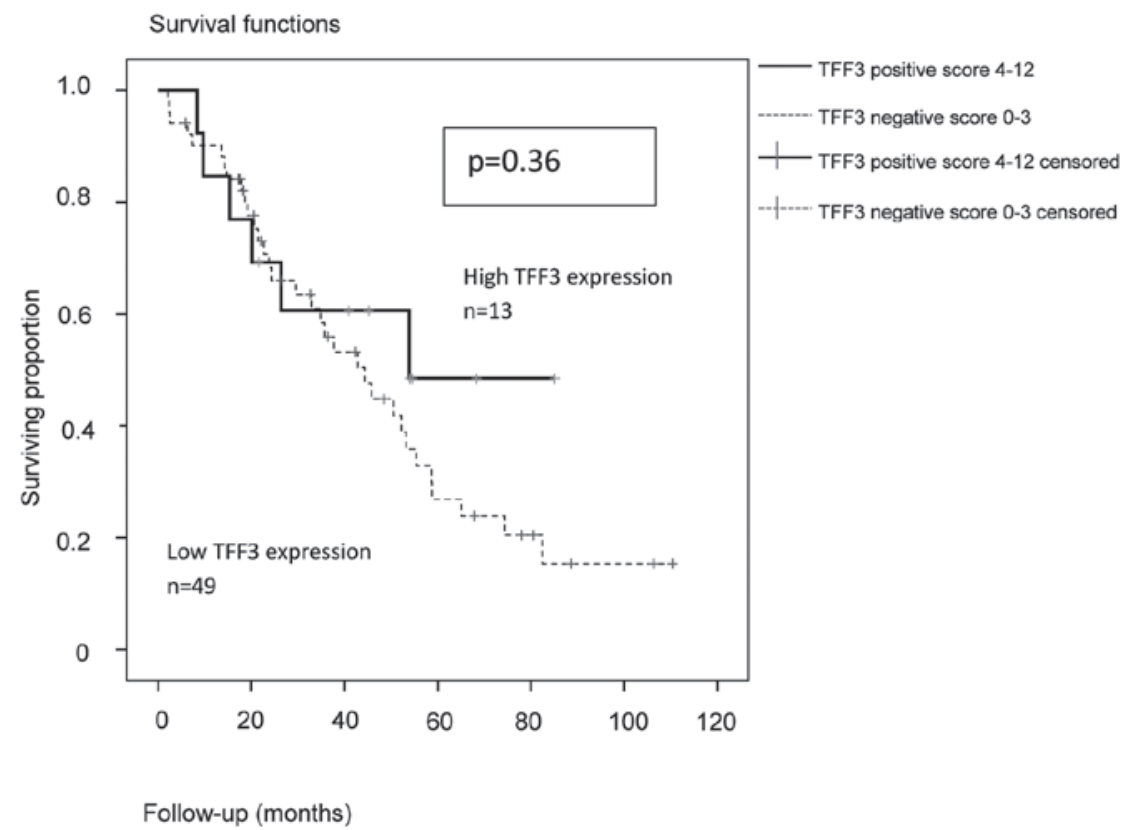

Figure 4. Kaplan-Meier analyses of overall survival was performed for the subgroup of 62 patients with high-grade serous ovarian cancer according to TFF3 expression (TFF3-positive vs. -negative). TFF3, trefoil factor 3.

Univariate Cox regression analysis. The univariate Cox regression analysis revealed a statistically significant effect of residual tumor on OS and PFS $(\mathrm{P}<0.001$ and $\mathrm{P}<0.001$, respectively) and of FIGO stage on OS and PFS ( $\mathrm{P}=0.001$ and $\mathrm{P}<0.001$, respectively). However, TFF3 positivity did not exert an effect on OS and PFS in the univariate Cox regression analysis ( $\mathrm{P}=0.158$ and $\mathrm{P}=0.156$, respectively) (Table III).
Multivariate Cox regression analysis. The multivariate Cox regression analysis for OS and PFS included age ( $>50$ vs. $\leq 50$ years), grade (G1 or G2 vs. G3), FIGO stage (I and II vs. III and IV), pathology (serous vs. others) and residual tumor (0 vs. $>0 \mathrm{~cm})$. Only FIGO stage and residual tumor exhibited a statistically significant correlation with poor $\mathrm{OS}(\mathrm{P}=0.041$ and $\mathrm{P}=0.043$, respectively). TFF3 expression was not found to be 
Table IV. Multivariate Cox regression analysis of standard variables among ovarian cancers for progression-free and overall survival.

A, Progression-free survival

\begin{tabular}{|c|c|c|c|c|}
\hline Variables & $\mathrm{P}$-value & HR & \multicolumn{2}{|c|}{$95 \%$ CI } \\
\hline Age, $>50$ vs. $\leq 50$ years & 0.458 & 1.310 & 0.642 & 2.672 \\
\hline Grade, G1 and G2 vs. G3 & 0.678 & 1.137 & 0.619 & 2.088 \\
\hline Histology, serous vs. other & 0.880 & 1.053 & 0.540 & 2.051 \\
\hline Residual tumor, 0 vs. $>0 \mathrm{~cm}$ & 0.041 & 0.511 & 0.269 & 0.971 \\
\hline FIGO stage, I and II vs. III and IV & 0.043 & 0.397 & 0.162 & 0.970 \\
\hline TFF3-negative (IRS 0-3) vs. -positive (IRS 4-12) & 0.105 & 1.673 & 0.898 & 3.118 \\
\hline
\end{tabular}

B, Overall survival

\begin{tabular}{llrr}
\hline Variables & P-value & HR & 95\% CI \\
\hline Age, $>$ 50 vs. $\leq 50$ years & 0.229 & 1.643 & 0.732 \\
Grade, G1 and G2 vs. G3 & 0.528 & 3.686 \\
Histology, serous vs. other & 0.645 & 1.243 & 0.632 \\
Residual tumor, 0 vs. >0 cm & 0.054 & 0.829 & 0.375 \\
FIGO stage, I and II vs. III and IV & 0.027 & 0.492 & 0.240 \\
TFF3-negative (IRS 0-3) vs. -positive (IRS 4-12) & 0.754 & 0.259 & 0.078 \\
\end{tabular}

TFF3, trefoil factor 3; FIGO, international federation of obstetrics and gynecology; HR, hazard ratio; CI, confidence interval.

significantly correlated with PFS or OS in all patients in the multivariate analysis $(\mathrm{P}=0.249)$ (Table IV).

\section{Discussion}

Ovarian carcinomas of different histological types originate from different precursor cells; thus, they retain specific gene and biomarker expression profiles (2). Ovarian cancer subtyping remains one of the major challenges in order to establish prognostic and predictive markers. Apart from histological subtype and the recently established binary grading system that forms subgroups of high-grade and low-grade serous EOC, very few predictive or prognostic biomarkers have been identified in order to better describe the clinical course of the disease, as well as sensitivity to different therapeutic approaches. Furthermore, genetic tumor profiling may reveal several prognostic and predictive genetic signatures. However, these signatures have yet to be validated. In this context, TFF3 was found to be involved in certain signatures.

The TFF family, which comprises three proteins (TFF1, TFF2 and TFF3), is involved in the development and progression of various types of cancers (12).

However, the role of TFF3 in ovarian cancer has not been fully elucidated (13). In breast cancer cells, TFF3 expression is generally positively associated with mammary carcinoma of the estrogen receptor-positive subtype, and TFF3 was recently identified as a promoter of tumor angiogenesis (4). In breast cancer, TFF3 expression is associated with poor survival, lymph node dissemination and distant metastasis. Furthermore, TFF3 may promote gastric cancer and its expression is associ- ated with increased microvessel density in gastric as well as breast cancer (4,5). High TFF3 mRNA levels in colorectal cancer tissues are associated with distant metastasis, recurrence and poor survival (12). Other authors investigated serum TFF3 levels in gastrointestinal cancer patients and found a correlation of high serum levels with advanced-stage disease and poor response to chemotherapy (12). TFF3 is an estrogen receptor-related gene in breast and ovarian cancer. Apart from the effect of TFF3 on endocrine therapy, TFF3 expression may also be associated with chemoresistance in breast cancer (14). As immunohistochemical staining of the estrogen receptor does not affect the clinical management of ovarian cancer, it is not part of the routine histopathological work-up of ovarian cancer. Thus, in the present study, we did not focus on the correlation of TFF3 expression with the estrogen receptor. However, further studies are required in order to elucidate the effect of simultaneous estrogen receptor and TFF3 expression in ovarian cancer.

This retrospective analysis of 91 patients with EOC shows that loss of TFF3 expression may be correlated with disease progression. To the best of our knowledge, this is the first report on the prognostic effect of TFF3 expression in this tumor entity.

Interestingly, in our patient collective, there was a trend for improved prognosis in TFF3-positive patients; however, this trend was not significant $(\mathrm{P}=0.151$ and $\mathrm{P}=0.155$ for $\mathrm{OS}$ and PFS, respectively). This tendency of differences in OS and PFS in TFF3-positive vs. -negative patients appears to be contradictory to the results of other tumor entities, which reported TFF3 to be a marker of poor prognosis (12). Above all, 
a correlation of higher TFF3 expression with poor prognosis was found in gastrointestinal tumors that were, in this respect, most intensively evaluated. Furthermore, TFF3 appears to be a potential marker for screening in this tumor entity. Although gastrointestinal tumors and EOC share certain common characteristics, TTF3 expression was hardly investigated in ovarian cancer. Only a very recent study demonstrated an effect of TFF3 expression on clinical course in EOC. The authors demonstrated a significant correlation of high TFF3 expression and lower risk of recurrence (15). However, that study by Jatoi et al (15) was designed to focus specifically on interactions between several potential prognostic markers, rather than analyze the impact of single markers. The results confirmed our observation of TFF3 as a protective marker against recurrence of EOC, in contrast to gastrointestinal tumors. Based on our results, two hypotheses may be conjured: TFF3-negative ovarian cancers may be more aggressive, regardless of stage and histopathological subtype; however, TFF3 positivity may be associated with higher chemosensitivity, which may explain the improved survival due to a better response to chemotherapy. In our patient collective, no significant correlation was observed between TFF3 expression and sensitivity to platinum agents. In order to elucidate this question, in vitro essays and further studies are required. In our patient collective, all histopathological subtypes were included. However, histological subtypes other than serous EOC were underrepresented. Under the assumption that TFF3 is part of the subtype markers for different histopathological entities of ovarian cancer, larger studies are required in order to clearly differentiate between the different histological subtypes (2). In particular, the subgroup of mucinous EOC should be further investigated due to its similarities to gastrointestinal tumors. Based on the dualistic model of ovarian carcinogenesis proposed by Kurman and Shih, we performed a subgroup analysis of high-grade serous ovarian cancers (type II tumors) (16). In our patient collective, the subgroup analyses of high-grade serous ovarian cancers yielded identical results. However, these results must be interpreted with caution, as the differences in OS and PFS between TFF3-positive and -negative patients were not significant.

A significant difference in TFF3 expression based on grade (G1 or 2 vs. G3) was observed, namely G3 tumors were less likely to express TFF3 ( $\mathrm{P}=0.05)$. To the best of our knowledge, there are no reported data on a correlation between grade and TFF3 overexpression to date. Further studies with larger patient collectives and subgroup analyses are required, as our patient collective was heterogeneous regarding histological subtype, stage and therapy.

\section{Acknowledgements}

We would like to thank Samira Adel and Katherina Kourtis for expert technical assistance. Furthermore, we would like to thank Prof. Dr. Dr. M. L. Hansmann Senckenberg (Institute of Pathology, University of Frankfurt), for providing the FFPE tissue samples.

\section{References}

1. Hennessy BT, Coleman RL and Markman M: Ovarian cancer. Lancet 374: 1371-1382, 2009.

2. Köbel M, Kalloger SE, Lee S, Duggan MA, Kelemen LE, Prentice L, Kalli KR, Fridley BL, Visscher DW, Keeney GL, et al: Biomarker-based ovarian carcinoma typing: A histologic investigation in the ovarian tumor tissue analysis consortium. Cancer Epidemiol Biomarkers Prev 22: 1677-1686, 2013.

3. du Bois A, Reuss A, Pujade-Lauraine E, Harter P, Ray-Coquard I and Pfisterer J: Role of surgical outcome as prognostic factor in advanced epithelial ovarian cancer: A combined exploratory analysis of 3 prospectively randomized phase 3 multicenter trials: By the arbeitsgemeinschaft gynaekologische onkologie studiengruppe ovarialkarzinom (AGO-OVAR) and the groupe d'Investigateurs Nationaux Pour les Etudes des cancers de l'Ovaire (GINECO). Cancer 115: 1234-1244, 2009.

4. Lau WH, Pandey V, Kong X, Wang XN, Wu Z, Zhu T and Lobie PE: Trefoil factor-3 (TFF3) stimulates de novo angiogenesis in mammary carcinoma both directly and indirectly via IL-8/CXCR2. PLoS One 10: e0141947, 2015.

5. Xiao P, Ling H, Lan G, Liu J, Hu H and Yang R: Trefoil factors: Gastrointestinal-specific proteins associated with gastric cancer. Clin Chim Acta 450: 127-134, 2015.

6. Kalloger SE, Köbel M, Leung S, Mehl E, Gao D, Marcon KM, Chow C, Clarke BA, Huntsman DG and Gilks CB: Calculator for ovarian carcinoma subtype prediction. Mod Pathol 24: 512-521, 2011.

7. Engels K, Knauer SK, Metzler D, Simf C, Struschka O, Bier C Mann W, Kovács AF and Stauber RH: Dynamic intracellular survivin in oral squamous cell carcinoma: Underlying molecular mechanism and potential as an early prognostic marker. J Pathol 211: 532-540, 2007.

8. Silverberg SG: Histopathologic grading of ovarian carcinoma: A review and proposal. Int J Gynecol Pathol 19: 7-15, 2000.

9. Tavassoli FA and Devilee P (Eds): Tumours of the ovary and peritoneum. In: Pathology and Genetics of Tumours of the Breast and Female Genital Organs. IARC Press, Lyon 2003.

10. Remmele W and Stegner HE: Recommendation for uniform definition of an immunoreactive score (IRS) for immunohistochemical estrogen receptor detection (ER-ICA) in breast cancer tissue. Pathologe 8: 138-140, 1987 (In German).

11. NCCN Clinical Practice Guidelines in Oncology (NCCN Guidelines ${ }^{\circledR}$ ): Ovarian Cancer Including Fallopian Tube Cancer and Primary Peritoneal Cancer. Version 2, 2011. https://www. tri-kobe.org/ncen/guideline/gynecological/english/ovarian.pdf. Accessed July 1, 2016.

12. Morito K, Nakamura J, Kitajima Y, Kai K, Tanaka T, Kubo H, Miyake $\mathrm{S}$ and Noshiro $\mathrm{H}$ : The value of trefoil factor 3 expression in predicting the long-term outcome and early recurrence of colorectal cancer. Int J Oncol 46: 563-568, 2015.

13. Walker G, MacLeod K, Williams AR, Cameron DA, Smyth JF and Langdon SP: Estrogen-regulated gene expression predicts response to endocrine therapy in patients with ovarian cancer. Gynecol Oncol 106: 461-468, 2007.

14. Chen Y, Chen C, Yang B, Xu Q, Wu F, Liu F, Ye X, Meng X, Mougin B, Liu G, et al: Estrogen receptor-related genes as an important panel of predictors for breast cancer response to neoadjuvant chemotherapy. Cancer Lett 302: 63-68, 2011.

15. Jatoi A, Vierkant RA, Hawthorne KM, Block MS, Ramus SJ, Larson NB, Fridley BL and Goode EL: Clinical and emergent biomarkers and their relationship to the prognosis of ovarian cancer. Oncology 90: 59-68, 2016.

16. Kurman RJ and Shih IeM: The dualistic model of ovarian carcinogenesis: Revisited, revised and expanded. Am J Pathol 186: 733-747, 2016. 